\title{
Can ultrasonography be useful in the diagnosis of urethral disorders of female-to-male transsexuals?
}

\author{
Jakub Krukowski, Adam Kałużny, Marcin Matuszewski \\ Department of Urology, Medical University of Gdańsk, Gdańsk, Poland
}

\begin{abstract}
Sonourethrography (SUG) is one of the basic imaging tools in the diagnosis of male anterior urethral strictures. So far, no one has described the use of this examination in transgender patients after "female-to-male" operation. Based on the presented case, we describe ultrasonographical features of the construction of a neophallus and offer information allowing a more accurate evaluation of urethral disorders in this group of patients.
\end{abstract}

Keywords: urethral stricture; ultrasonography; sonourethrography; female-to-male transsexualism

\section{Introduction}

Phalloplasty is the standard of care for female-tomale transgenders [1]. One of the most common complication after these operations, alongside urethrocutaneus fistulas, are urethral strictures, reaching up to $58 \%$ of patients. Reasons may be an insufficient vascular supply of the local flaps and the inappropriate width of the phallic urethra [2]. The best treatment option, as for men with recurrent urethral strictures, is urethroplasty [3]. For better planning of the operation, imaging examinations are required to show number, localization, and length of the damaged urethra. Usually, cystourethrography (CUG) is performed, but sonourethrography (SUG) may be an alternative for this imaging study.

Received 04.02.2019 Accepted 19.03.2019

Med Ultrason

2019, Vol. 21, No 3, 359-361

Corresponding author: Jakub Krukowski, MD

Department of Urology, Medical University of Gdańsk, Gdańsk, Poland; Smoluchowskiego

17 St., 80-952, Gdańsk, Poland

E-mail: jakub.i.krukowski@gmail.com

\section{Case report}

A 27-year-old man visited the outpatient clinic because of escalated problems with micturition. He reported a weak, continuous urinary stream, with no hesitancy, nocturia or urgency. In 2014 the patient had underwent a "female-to-male" sex change operation with a total phalloplasty with a latissimus dorsi musculocutaneous flap [4].

Physical examination showed no abnormalities. Uroflowmetry had, typical for the urethral stricture, flat, "box shape" curve. Maximal flow rate (Qmax) was $7.7 \mathrm{ml} / \mathrm{sec}$. The transabdominal ultrasonography (US) did not reveal any changes in the urinary system or post-void residual urine, so the decision for SUG was taken.

The technique of the urethral SUG was similar to proposed by McAninch et al [5]. The urethra was dilated with a saline solution. Because of the localization of the urethral stricture close to the meatus, we used an $18 \mathrm{G}$ peripheral venous catheter instead of a Foley catheter. US evaluation was performed with a BK Medical Flex Focus 800 equipped with a $18 \mathrm{~L} 5$ linear transducer operated at a transmission frequency from 9 to $12 \mathrm{MHz}$. SUG revealed 


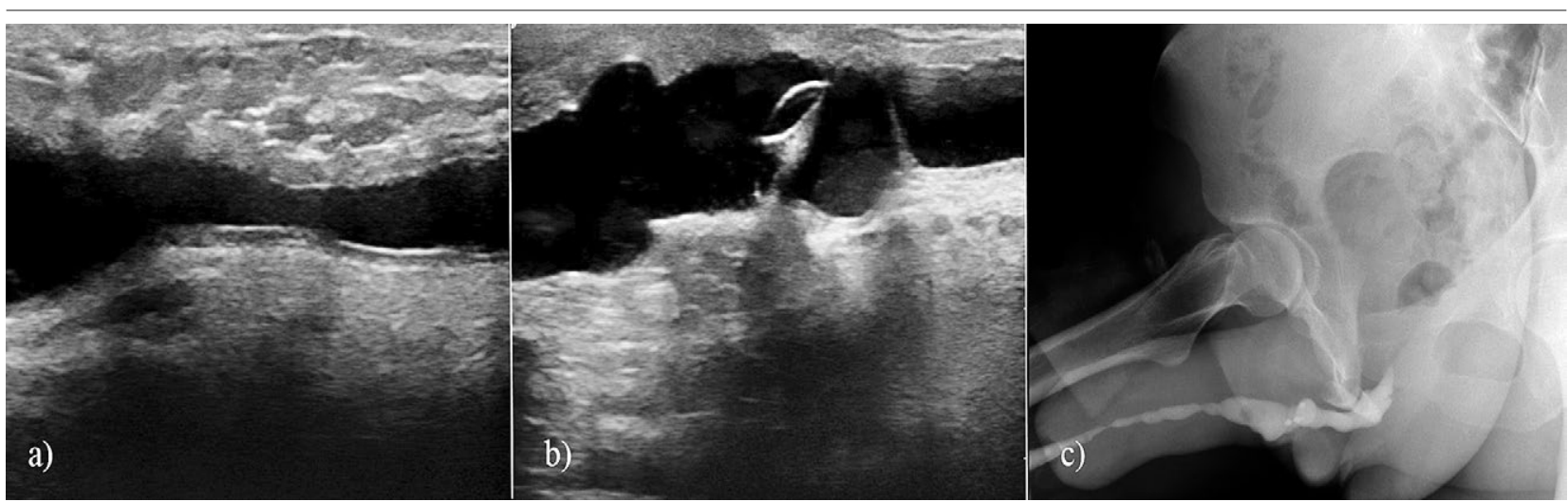

Fig 1. a) Ultrasound aspect of the urethral stricture in the phallic part; b) ultrasound aspect of the anastomotic part of the urethra. In the central part of the picture are visible hairs with microcalcifications; c) retrograde cystourethrography. In the penile urethra two critical strictures are visible. Additionally, in the area of the anastomotic part of neourethra are visible elements of a hydraulic penile prosthesis.

two strictures localized in the phallic urethra. The first was $10 \mathrm{~mm}$ behind the meatus (12 $\mathrm{mm}$ length), and the second in the central part of neophallus (23 mm length) (fig 1a). Urethral epithelium in the damaged urethra was hyperechogenic in comparison to the healthy portion. Additionally, in the anastomotic part of the urethra, we revealed hairs with calcifications on their surface (fig 1b). The CUG confirmed the SUG findings (fig 1c).

The patient was qualified for urethroplasty.

\section{Discussions}

Our paper is the first that describes the US evaluation of anterior urethra in "female-to-male" transgender after the sex-change operation.

Similarly to men with urethral strictures, retrograde and voiding CUG are the examinations of choice. However, the neourethra has no division typical for the male urethra (table I). The most common localization of strictures is the area of connection between a fixed and phallic part, found in over $40 \%$ of all diagnosed stenoses in this group of patients [3].

Due to the specificity of organ formation, it is not possible to compare the US structure of neophallus with a normal penis. Depending on the used technique,

Table I. The division of the urethra in transgender patients and corresponding sections of the normal male urethra [3].

\begin{tabular}{ll}
\hline Transsexual urethra & Male urethra \\
\hline Native urethra & Prostatic urethra \\
Fixed part & Membranous urethra \\
Anastomotic part & Bulbar urethra \\
Phallic part & Penile urethra \\
Meatus & Meatus \\
\hline
\end{tabular}

the width of the organ varies from 35 to $120 \mathrm{~mm}$ [6]. In comparison to the normal penile urethra, neourethra is localized deeper in the penile body. In our patient, the length between the skin and urethral lumen was around $9-10 \mathrm{~mm}$ (in contrast, for healthy men it is 3 to $5 \mathrm{~mm}$ ). Technically, this means the need for using lower frequencies during the examination.

The US image of neophallus is dominated by the fatty tissue surrounding the neourethra. Additionally, in the dorsal part of the organ, the elements of the hydraulic penile prosthesis are usually placed. Also, the urethra has no typical structure. The most significant difference is the lack of a corpus spongiosum. It makes it impossible to assess the periurethral fibrosis (in case of a normal penis visible as a hyperechogenic structure surrounding the damaged part of the urethra), which is one of the main factors determining the extent and type of urethroplasty. In this situation, it may be helpful to measure the length of the changed urethral surface. The damaged urethral epithelium is visible as a hyperechogenic linear structure, noticeably delimited from the healthy part of the urethra.

In the literature, there is a lack of information about the "physiological" size of the neourethra. Some indication might be the fact that the width of the flap used to form the urethra is from 30 to $35 \mathrm{~mm}$, so its minimum diameter should be about 9-10 $\mathrm{mm}[7,8]$.

In conclusion, despite many similarities to the normal penis, neophallus and neourethra evidence an utterly different structure. It determines the specific image on US. The technique of examination in both groups of patients is similar, however, the degree and extent of urethral damage in transgender patients can be estimated only in the measurement of the length of the stenosed part, without assessing the fibrosis of the surrounding tissues. 


\section{References}

1. Monstrey S, Vercruysse H, De Cuypere G. Is gender reassignment surgery evidence based? Recommendation for the seventh version of WPATH Standard of Care. Int J Transgender 2009;11:206-214.

2. Rohrmann D, Jakse G. Urethroplasty in female-to-male transsexuals. Eur Urol 2003;44:611-614.

3. LumenN, MonstreyS, GoessaertAS, OosterlinckW,Hoebeke P. Urethroplasty for strictures after phallic reconstruction: a single-institution experience. Eur Urol 2011;60:150-158.

4. Jun MS, Pušica S, Kojovic V, et al. Total Phalloplasty With Latissimus Dorsi Musculocutaneous Flap in Female-tomale Transgender Surgery. Urology 2018;120:269-270.
5. McAninch JW, Laing FC, Jeffrey RB Jr. Sonourethrography in evaluation of urethral stricture: preliminary report. J Urol 1988;139:294-297.

6. Morrison SD, Shakir A, Vyas KS, Kirby J, Crane CN, Lee GK. Phalloplasty: A Review of Techniques and Outcomes. Plast Reconstr Surg 2016;138:594-615.

7. van der Sluis WB, Smit JM, Pigot GLS, et al. Double flap phalloplasty in transgender men: Surgical technique and outcome of pedicled anterolateral thigh flap phalloplasty combined with radial forearm free flap urethral reconstruction. Microsurgery 2017;37:917-923.

8. Gilbert DA, Schlossberg SM, Jordan GH. Ulnar forearm phallic construction and penile reconstruction. Microsurgery $1995 ; 16: 314-321$. 